\title{
Paysages funéraires de l'âge du Bronze
}

\author{
José Gomez de Soto
}

\section{OpenEdition}

\section{Journals}

Édition électronique

URL : http://journals.openedition.org/rao/2219

DOI : $10.4000 /$ rao.2219

ISBN : 978-2-7535-3432-2

ISSN : 1775-3732

Éditeur

Presses universitaires de Rennes

Édition imprimée

Date de publication : 25 décembre 2013

Pagination : 311-312

ISBN : 978-2-7535-3430-8

ISSN : 0767-709X

\section{Référence électronique}

José Gomez de Soto, «Paysages funéraires de l'âge du Bronze », Revue archéologique de l'Ouest [En ligne], 30 | 2013, mis en ligne le 25 décembre 2013, consulté le 04 décembre 2020. URL : http:// journals.openedition.org/rao/2219; DOI : https://doi.org/10.4000/rao.2219

Ce document a été généré automatiquement le 4 décembre 2020.

Tous droits réservés 


\title{
Paysages funéraires de l'âge du Bronze
}

\author{
José Gomez de Soto
}

\section{RÉFÉRENCE}

Bérenger D., Bourgeois J., Talon M. et Wirth S. (éd.), 2012 - Gräberlandschaften der Bronzezeit. Paysages funéraires de l'âge du Bronze (actes du colloque international d'Herne, 15-18 octobre 2008), Bodenaltertümer Westfalens 51, Darmstadt, Ph. V. Zabern, 2012, 700 p. (ISBN 978-3-8053-4511-8)

1 Du 15 au 18 octobre 2008 s'est tenu au musée de Herne en Westphalie un colloque international intitulé «Gräberlandschaften der Bronzezeit. Paysages funéraires de l'âge du Bronze ». Cette manifestation s'insère dans une succession de colloques bi-annuels inaugurée par celui sur le RSFO tenu à Nemours en 1986. Les actes, qui viennent de paraître, réunissent trente-sept contributions, rédigées en allemand, en français ou en anglais. Les résumés, donnés dans les trois langues, permettront à ceux qui ne maîtrisent pas l'une ou l'autre des langues de tirer le meilleur parti de cet ouvrage. On regrettera la valeur inégale de ces résumés, quelques-uns restant fâcheusement trop peu informatifs. Il ne saurait évidemment être question ici de rendre compte de chacun des articles ; nous nous bornerons donc à un choix, par définition arbitraire.

2 Le premier thème du colloque est intitulé " Analyse des nécropoles appuyées par SIG » (3 contributions). F. Delrieu et P.-Y. Milcent traitent des tumulus du Cézalier et du Causse noir. Dans les deux régions du Massif central, les nécropoles tumulaires obéissent aux mêmes modalités d'implantation et de dynamique. Pour le centre-ouest de la Bretagne, M. Fily, A. Villard-Le Tiec, Y. Menez et T. Lorho montrent l'importance du choix des hauteurs. L'utilisation du LIDAR fournit d'importantes informations et la nécropole de Paule donne lieu à une présentation. L'article de J. May et T. Hauptmann (p. 77-104) replace le célèbre tumulus « royal » de Seddin en Allemagne du Nord, dans un contexte qui n'est pas sans préfigurer celui des sites princiers du Hallstatt final et de 
La Tène ancienne, un cas exceptionnel pour l'âge du Bronze, comme le rappellera $\mathrm{H}$. Thrane dans sa conclusion.

3 Le second thème est consacré aux «Éléments d'organisation spatiale à l'intérieur des nécropoles " (9 contributions). Sont traités les cas particuliers de Große Heide en Lünebourg (M. Geschwinde); de la Normandie, culturellement entre Armorique et Wessex, dans le cadre du projet de recherche en cours sur la presqu'île de la Hague (F. Delrieu et S. Quévillon); du Gâtinais au BF I-IIa/Bz D-Ha A1 (H. Froquet-Uzel et E. Trébuchet, qui produisent de précieux documents inédits sur une région mal connue); le complexe cimetière à enclos fossoyés des âges du Bronze et du Fer de Borken-Gemenwirthe en Westphalie (C. Kersten). La présentation de la nécropole de Destelbergen/Eenbeekeinde dans les Flandres belges par G. De Mulder et E. Deweirdt, croisant SIG et un grand nombre de dates $\mathrm{C}^{14}$, donne lieu à une belle étude diachronique.

4 J. E. Fries, B. Herring et $\mathrm{O}$. M. Wilbertz font le point sur les nécropoles à longs tumulus et enclos en trou de serrure. M. David-Elbiali développe un remarquable discours sur les paysages funéraires du genre et les rôles sociaux, à partir d'exemples pris au nord et au sud des Alpes présentés dans leur diversité spatio-temporelle et confrontés aux données des habitats et des dépôts. L'éclairage par les sources homériques, qu'on tend trop souvent à négliger, est judicieusement exploité. A. Hänel s'interroge sur la symbolique des orientations des ouvertures des mégalithes de l'Europe du Nord à l'Armorique. I. Kerouanton, à propos du site du Champ-des-Rochers à Soyaux en Charente, pose judicieusement la question de la signification des enclos fossoyés protohistoriques sans sépulture, et présente de nouveaux cas de "sépultures » assises laténiennes.

5 Le troisième thème aborde les continuités et discontinuités des espaces funéraires au cours du temps (17 contributions). Les questions régulièrement abordées sont celles du passage plus ou moins généralisé, mais loin d'être absolu, de la pratique (et pas du rite, le mot bien que d'usage courant, est impropre dans le cas présent) de l'inhumation à celle de l'incinération entre le Bronze moyen et le Bronze final, celle de la continuité ou de la reprise des nécropoles, avec un questionnement paléodémographique. Les études portent sur des régions plus ou moins vastes ou des sites particuliers. J. De Reu, $\mathrm{J}$. Hammond, S. Toron et J. Bourgeois traitent dans une étude ambitieuse la Belgique, le Nord de la France et le Sud-Est de l'Angleterre, avec un intérêt particulier porté aux enclos circulaires. D'autres études concernent la France : le tronçon seine-et-marnien de la vallée de la Seine (V. Delattre et R. Peake), l'Alsace, la Lorraine et la Champagne (M.-P. Koenig, S. Plouin, J. Vanmoerkerke, C. Veber), l'Yonne (F. Muller et M. Roscio), la nécropole de Jâlons dans la Marne (S. Lenda, G. Deborde, G. Depierre, I. Turé).

6 L'Allemagne du Nord est copieusement illustrée par des études consacrées à la région Elbe-Weser-Dreicke (K.P.Hofmann) et aux cimetières de Schafberg (J. Kaiser et T. Puttkammer), Liebersee (W. Ender). En République tchèque, M. Ernée présente l'évolution tempo-spatiale de la nécropole unéticienne de Prague-Miškovice depuis sa fondation au Néolithique, J. Peška celle d'Hulin.

7 Certaines études spécialisées sur des phénomènes particuliers méritent d'être signalées. G. Manschus se consacre aux tombes à hochets, dans une contribution centrée sur la culture de la Lusace en Saxe, mais dans une perspective européenne. C. Kacsó, C. Metzner-Nebelsick, L. D. Nebelsick s'interrogent sur les élites de Roumanie du Nord, à propos d'un grand tumulus et d'un monument cultuel livrant des traces de 
sacrifices considérées comme liées au culte des ancêtres. K. Malek et M. A. OčirGorjaeva présentent une tombe à char "pauvre » de la civilisation des Catacombes, s'interrogeant sur son éventuelle fonction plutôt rituelle que significative du statut social. Enfin, D. Brandherm revient de manière critique sur l'hypothèse d'un lien entre une possible catastrophe climatique et les transformations des sociétés du Bronze final au milieu du $\mathrm{XII}^{\mathrm{e}}$ siècle, et privilégie, avec une solide argumentation, les processus internes d'évolution de ces sociétés.

8 Le quatrième thème est réservé aux "paysages sacrés ", c'est-à-dire à la place des espaces funéraires dans le paysage (6 contributions). Q. Bourgeois et D. Fontijn s'interrogent à propos de la "diversité dans l'uniformité » et de l'« uniformité dans la diversité " des monuments des nécropoles tumulaires des Pays-Bas, et de la signification sociale du phénomène dans l'espace et la longue durée. H. Fokkens, à partir des tumulus d'Oss aux Pays Bas, constatant que les ensembles de tumulus peuvent n'être utilisés que sur un temps relativement court, interprète leur devenir comme celui d'un lieu de culte des ancêtres, auxquels l'agrégation de nouvelles sépultures, parfois après de longs délais de latence serait une forme de revendication de pouvoir sur un territoire. Une réflexion qui pourrait s'appliquer à un grand nombre des nécropoles françaises, à enclos fossoyés et autres, qui montrent les mêmes discontinuités.

$9 \quad$ L'interprétation du karst charentais de La Rochefoucauld en paysage rituel de la culture des Duffaits proposée par S. Manem, sans doute séduisante, fait quelque peu figure de vision romantique du fait d'évidentes faiblesses: absence de confrontation entre données de grottes présentées comme lieux de culte et/ou de rassemblement et données d'habitats au sein de la culture des Duffaits, à l'évidence le problème majeur pour le débat; datation maintenant discutable du site du Fouilloux ; etc.

10 L'ambitieuse contribution de C. Marcigny tire le meilleur parti des apports récents de l'archéologie, et tout particulièrement préventive, en Normandie. Trois niveaux d'échelles funéraires et territoriales sont définis : le territoire immédiat; le terroir/ espace «biologique»; la chefferie, particulièrement bien illustrée par le cas de la Hague.

11 M. Freudenberg étudie en détail le paysage funéraire de la région de Hüsby en Allemagne du Nord, principalement illustré par une tombe aristocratique et le monument cultuel associé. La tombe paraît celle d'un personnage hors du commun à dimension « internationale », entretenant des liens avec plusieurs aires cultuelles.

12 Particulièrement intéressante est l'étude menée sur la longue durée en Basse-Lusace par E. Bönisch, qui permet de suivre l'évolution, d'un habitat dispersé et de groupes familiaux de tumulus au $\mathrm{Bz} C$ et au $\mathrm{Bz} \mathrm{D}$, aux villages et ensembles funéraires plus vastes du Ha B. De vastes nécropoles ayant été utilisées tout au long de la période lusacienne existent parallèlement.

13 A. Jockenhövel et $\mathrm{H}$. Thrane concluent les actes en synthétisant les données présentées et ouvrant des pistes de recherche, le premier en traitant de la question des interactions et identités régionales au cours de l'âge du Bronze, le second en présentant une réflexion générale sur les tumulus.

14 Comme on peut en juger par ce trop bref compte-rendu de ses actes, les quatre thèmes du colloque, inévitablement, se chevauchent, donnant parfois l'impression d'un classement un peu arbitraire des différentes contributions. Broutille, ne boudons pas 
notre satisfaction de disposer d'une telle richesse d'informations et des fructueuses pistes de réflexion ouvertes! Incontestablement, un volume maintenant essentiel pour la compréhension des sociétés de l'âge du Bronze, et même pour certaines régions, de l'âge du Fer.

15 Dans le domaine de l'archéologie spatiale protohistorique, la recherche française accusait un certain retard par rapport à celle de nos collègues européens. Ce retard commence à largement se combler, comme le montre la part appréciable prise par les chercheurs français - dont ceux de l'UMR 656 «CReAAH » - à ce colloque. Personne ne s'en plaindra!

\section{AUTEURS}

\section{JOSÉ GOMEZ DE SOTO}

Directeur de recherche émérite au CNRS, UMR 6566 « CReAAH » 